\title{
Investigating Body Mass Index and Body Composition in Patients with Schizophrenia: A Case-Control Study
}

\author{
M. Marthoenis, ${ }^{1}$ M. Martina, ${ }^{1}$ Rudi Alfiandi, ${ }^{1}$ D. Dahniar, ${ }^{1}$ Rini Asnurianti, ${ }^{1}$ Hasmila Sari, ${ }^{1}$ \\ Jacqueline Nassimbwa, ${ }^{2}$ and S. M. Yasir Arafat ${ }^{3}$ \\ ${ }^{1}$ Department of Psychiatry and Mental Health Nursing, Universitas Syiah Kuala, Banda Aceh, Indonesia 23111 \\ ${ }^{2}$ Health Plus Development Communication, Kampala, Uganda \\ ${ }^{3}$ Department of Psychiatry, Enam Medical College and Hospital, Dhaka 1340, Bangladesh
}

Correspondence should be addressed to S. M. Yasir Arafat; arafatdmc62@gmail.com

Received 16 December 2021; Revised 24 January 2022; Accepted 18 February 2022; Published 27 February 2022

Academic Editor: Anna Comparelli

Copyright ( $) 2022$ M. Marthoenis et al. This is an open access article distributed under the Creative Commons Attribution License, which permits unrestricted use, distribution, and reproduction in any medium, provided the original work is properly cited.

\begin{abstract}
Background. Antipsychotics exert metabolic side effects, and prolonged treatment with antipsychotics causes changes in body weight and muscle composition. Nevertheless, reports on the changes in body composition of patients with schizophrenia have been limited. This study is aimed at comparing the body mass index and body composition of patients with schizophrenia with healthy individuals in Indonesia. Methods. A total of 195 patients with schizophrenia (148 males and 47 females) and 195 healthy individuals matched by gender were recruited. Using the Bioelectrical Impedance Analysis method, the participants' body compositions were measured. Results. Compared to healthy individuals, the patient group exhibited a higher rate of underweight as well as a lower rate of overweight and obesity. Multiple regression analysis confirmed the associations between the body mass index and all measured body compositions. Furthermore, the diagnosis of schizophrenia is significantly associated with lower muscle mass, lower bone mass, higher basal metabolic rate, older metabolic age, and higher total body water. Conclusions. The results showed that patients with schizophrenia are at a greater risk of a lower quality of certain components of body composition. Priority should be given to research that addresses increasing the patient's level of physical activity.
\end{abstract}

\section{Introduction}

Obesity is more prevalent in patients with schizophrenia than in the general population $[1,2]$, even after controlling for age, gender, and psychiatric practice attended [3]. Obesity is also an independent risk factor for multiple chronic conditions, including cardiovascular diseases, diabetes, hypertension, and stroke [4-6]. Among patients with schizophrenia, the significant predictors of obesity include gender, education level, smoking behavior, type 2 diabetes, a higher level of triglycerides [7], and antipsychotic medication [8].While a patient's body mass index (BMI) is a marker for their nutritional status, it does not reflect the changes in their body composition. The consideration of detail of body composition is significant because, although BMI is significantly correlated with fat mass, the value can be misleading depending on the individual level of adiposity [9].
In recent years, the Bioelectrical Impedance Analysis (BIA) method has been more frequently used to measure body composition variables. BIA is a better indicator of obesity than BMI in patients with schizophrenia [10]. It works with the principle that the transit time of a low-voltage electric current through the body depends on the characteristics of individual body composition [11]. While the dualemission X-ray absorptiometry (DXA) is the contemporary reference method for the body composition assessment, as it has proven more accurate body composition components $[12,13]$, accessibility to this sophisticated measure is not always feasible. Using portable BIA equipment is preferable in this study because it is invasive, inexpensive, relatively quick to operate, and suitable for examining a large number of subjects.

Several studies using the BIA have been conducted measuring the body composition of patients with schizophrenia. 
A hospital-based study found that male patients with schizophrenia under antipsychotic treatments have more body fat, a higher percentage of fat, lower muscle mass, and lower body water compared to the healthy controls, while the female patients have a significantly lower percentage of body fat, higher muscle mass, and higher body water compared to the health controls [9]. Furthermore, a community-based survey found that individuals with schizophrenia tend to have abdominal obesity, higher fat percentage, and lower muscle mass [14]. Another study found that the body fat was associated with height, weight, BMI, and abdominal and waist circumference, while the basal metabolic rate was associated with age, height, weight, abdominal, and waist circumference among patients with schizophrenia [15]. The inconsistency of factors associated with components of body composition between studies demands further investigations.

While BMI studies have been conducted among patients with mental health disorders in Indonesia [16-18], body composition studies have not been conducted. The proximation of obesity or body fat among patients with schizophrenia in the country may therefore be erroneous with available data on BMI, and management protocols may not be well informed. The main objective of this study was to measure BMI and body composition (using BIA) among psychiatric inpatients with schizophrenia and compare them with healthy controls. To the best of our knowledge, this is the first targeted study ever conducted in Indonesia. Based on the findings of previous related studies and theories, we hypothesize that the patients with schizophrenia have higher BMI, total body water, and body fat but lower muscle mass, bone mass, physique rating, and basal metabolic rate compared to the healthy controls.

\section{Methods}

2.1. Study Subjects. The subjects were 195 inpatients (148 males and 47 females) diagnosed with schizophrenia based on DSM-IV diagnostic criteria at the Aceh Psychiatric Hospital in Indonesia. The subjects' diagnoses were obtained from their medical records. As a reference group, 195 healthy volunteers were also included. The reference group was recruited from the general population using convenience sampling methods. Before inclusion into the study, the volunteers were screened with a semistructured interview to ensure the absence of both psychiatric disorders and the use of antipsychotics. The semistructured interview consists of questions whether they had ever been diagnosed with any mental disorder or had the history of antipsychotic medication use or think they had experienced any psychiatric symptoms. Only volunteers who confirm that they never had any of those problem were included in the study. For both the patients and volunteers, we also asked about their age, marital status, education, and occupation. The financial status was examined by their occupation status, whether they had bank account and savings, either at the bank or elsewhere. Data collection was conducted between June and August 2020. The study was approved by an ethics committee at Universitas Syiah Kuala, and all participants pro- vided informed consent before participating in the study. Permit for data collection among patients with schizophrenia was also obtained from the psychiatric hospital management.

2.2. Procedure. The subjects' demographic information and medications were compiled from their medical records, and the patients' antipsychotic medication was converted to a chlorpromazine equivalent [19]. Body composition was measured with the Tanita RD-953 analyzer [20]. This measurement procedure requires the subject to stand barefooted on the analyzer, while the height, date of birth, and level of activity were entered into the device. The device then calculates their weight, BMI, body fat percentage, muscle mass, muscle quality score, physique rating, bone mass, visceral fat, basal metabolic rate, metabolic age, and total body water. In addition, the blood pressure was measured using electronic blood pressure equipment while in a sitting position.

The body fat percentage was defined as the proportion of fat to the total body weight. Muscle mass is the predicted weight of muscle in the body, which includes the skeletal muscle, smooth muscles, and water contained in the muscles. Muscle quality score is calculated by the amount of muscle mass against the individual height. The larger score, the more muscle mass an individual has. Physique rating measures the muscle and body fat level and is rated as one of nine body types. Bone mass predicts the weight of bone minerals in the body. Visceral fat explains the amount of fat in the abdominal area to protect the vital organs. Basal metabolic rate is the daily minimum level of energy or calories that the body needs when at rest to function effectively. The metabolic age is the comparison of individual basal metabolic rate to the average basal metabolic rate of the chronological age group. Total body water is the amount of fluid in the individual body [21]. Furthermore, BMI was categorized as the following: underweight $(\mathrm{BMI}<18.5)$, normal $(18.5 \geq \mathrm{BMI}<24.9)$, overweight $(25 \geq \mathrm{BMI}<27)$, and obese $(\mathrm{BMI} \geq 27)$.

2.3. Statistical Analysis. Demographic and clinical characteristics were analyzed using descriptive statistics indicating the number and percentage, mean and standard deviation (SD), or median and interquartile range (IQR). The differences in demographic and clinical variables between the patients and the control group were performed using the Chisquared test for categorical data and independent sample $t$ -test analyses for continuous data. The independent sample $t$-test was also used to calculate the mean differences in body composition between the groups. Furthermore, multiple linear regression analysis was performed to predict demographic and other variables that are independently associated with each component of body composition. The independent variables associated with the components of body composition were included in the multiple linear regression analysis. Only variables with a significant $p$ value $(<0.05)$ were reported. Data analysis was carried out using the STATA 13 statistical software [22]. 


\section{Results}

3.1. Demographics. The mean (SD) age of all participants was 34 years (7.5), and the patients with schizophrenia were older, on average (36 years) (0.6), than the control group (32 years) $(0.6)(p<0.001)$. More than half $(63.8 \%)$ of the control group was married, while only approximately onethird $(36.2 \%)$ of the patients were married $(p<0.001)$. The vast majority of the patients only graduated from elementary school (98.3\%), whereas the majority of the control group had a university education level (95.2\%). Furthermore, the vast majority of the patient's group were jobless or not working at the moment $(80 \%)$, did not have bank account (97.4\%), and did not have any savings, either at the bank or somewhere else $(86 \%)$, indicating a low socioeconomic status of the patient group. No difference in systolic and diastolic blood pressures was observed. The median dosage of medication converted to a chlorpromazine equivalent was $475 \mathrm{mg}$ per day (IQR $=400-700)$. Detailed sociodemographic and clinical characteristics of the study participants are presented in Table 1.

3.2. Body Composition Measurements. Table 2 shows the differences in body composition between patients with schizophrenia and healthy controls. Overall, the patients with schizophrenia have a lower mean of body composition components compared to the healthy controls, except for metabolic age and total body water. The patients were shorter in height and lower in weight, BMI, muscle mass, bone mass, and basal metabolic rate $(p<0.001)$ compared to the healthy controls. In addition, their mean metabolic age was older $(p=0.03)$, and their total body water was higher than the control group $(p<0.001)$. A mean difference of body fat, muscle quality score, physique rating, and visceral fat between the groups was not observed $(p>0.05)$. The proportion of BMI categories between groups was also significantly different $(p<0.001)$. The patients' group has a higher underweight proportion but less prevalence in the overweight and obese groups. The details of the difference in BMI between patients and healthy controls are presented in Table 3.

3.3. Multiple Regression Analysis. Further statistical analysis confirms that BMI was associated with all measured body composition factors. BMI was associated with a higher body fat percentage, heavier muscle mass, better muscle quality, lower physique rating, higher bone mass, a larger amount of visceral fat, higher basal metabolic rate, older metabolic age, and lower total body water. Similar to BMI, gender was also associated with all components of the body composition, except with muscle quality and physique rating. Furthermore, schizophrenia was independently and significantly associated with lighter muscle mass, lower bone mass, higher basal metabolic rate, older metabolic age, and more total body water. Regular exercise was also significantly associated with a lower percentage of body fat, heavier muscle mass, better muscle quality, better physique rating, heavier bone mass, and lower visceral fat. Detailed regression analyses examining the association between the diagnosis of schizophrenia under antipsychotic treatments and body composition are presented in Table 4.

\section{Discussion}

This study examined the BMI and body composition of patients with schizophrenia compared with healthy controls. Patients undergoing antipsychotic treatment have a lower mean of muscle mass, bone mass, and basal metabolic rate while also exhibiting an older metabolic age and a higher percentage of body water compared to healthy subjects. Some of the findings in this study are in line with the previous reports, whereas others contradict the literature.

Previous studies have indicated that body fat mass and body fat percentage were higher in patients with schizophrenia than healthy individuals $[9,23]$. In contrast, this study suggests that both groups have an average body fat of $25 \mathrm{~kg}$, and no statistical difference was found between the groups. The lower muscle mass in the patients' group found in this study is in line with an earlier report [9], which stated that these findings are associated with several factors, including age and low physical activity [24]. Therefore, our findings are relevant to the treatment condition of the study population, as the vast majority of patients were locked in nursing wards and barely had any physical activity.

A relatively higher rate of underweight and a lower rate of overweight and obesity among the patient group compared to the normal controls were found in this study. The finding of $10.3 \%$ of patients with underweight has decreased from the $17 \%$ underweight rate reported in a previous study in the same setting [18], but it is still relatively higher than the rate of underweight in patients with schizophrenia reported in a recent meta-analysis (6.2\%) [25]. Nevertheless, the rate of overweight and obesity among the patients has changed from $8 \%$ and $5 \%$ reported earlier [18] to $7.7 \%$ and $18.5 \%$ found in this study, respectively. A lower rate of overweight and obese among inpatients with schizophrenia might be because of the majority of the patients come from low socioeconomic groups with financial difficulties. In other words, the patients had been poor and could not afford adequate food before hospitalization. During hospital treatment, the patients only eat food provided by the hospital and barely any snacks in between. Therefore, their body weight was most likely to be within normal weight. Also, the financial status of the patients, where the fast majority did not have any occupation, had no bank account, and had no savings, confirms their low socioeconomic status. Furthermore, following our earlier study dissemination, the psychiatric hospital management has made several changes concerning the meal and nutritional management of the patients. These changes include the employment of new nutritionists and the replacement of the food supplier, thus providing better services. The improvement of the prehospital financial condition of the patients might also be responsible for the higher rate of obesity during hospitalization.

The correlation between BMI and all body composition components measured is consistent with previous reports $[9,23,26]$. Despite BMI being a marker for nutritional status in patients with schizophrenia, it may not reflect changes in 
TABLE 1: Sociodemographic and clinical characteristics of participants $(n=390)$.

\begin{tabular}{|c|c|c|c|c|c|}
\hline Characteristics & Total & Schizophrenia & Control & $x 2$ or $t$ & $p$ value \\
\hline Age (years old) & $34 \pm 7.5$ & $36 \pm 0.6$ & $32 \pm 0.6$ & 4.8 & $0.001^{*}$ \\
\hline \multicolumn{4}{|l|}{ Marital status (\%) } & 36 & $0.001^{*}$ \\
\hline Unmarried & $177(45.4)$ & $118(66.7)$ & $59(33.3)$ & & \\
\hline Married & $213(54.6)$ & $77(36.2)$ & $136(63.8)$ & & \\
\hline \multicolumn{4}{|l|}{ Education } & 232.6 & $0.001^{*}$ \\
\hline Elementary (1-6 yrs) & $117(30.0)$ & $115(98.3)$ & $2(1.7)$ & & \\
\hline High school (7-12 yrs) & $126(32.3)$ & $73(57.9)$ & $53(42.1)$ & & \\
\hline University & $147(37.7)$ & $7(4.8)$ & $140(95.2)$ & & \\
\hline \multicolumn{4}{|l|}{ Occupation } & 199.2 & $0.001^{*}$ \\
\hline Jobless or not working & $95(24.4)$ & $76(80)$ & $19(20)$ & & \\
\hline Irregular job & $161(41.3)$ & $118(73.3)$ & $43(26.7)$ & & \\
\hline Formal job & $134(34.4)$ & $1(0.7)$ & $133(99.3)$ & & \\
\hline \multicolumn{4}{|l|}{ Having bank account } & 343.5 & $0.001^{*}$ \\
\hline Yes & $197(50.5)$ & $7(3.6)$ & $190(96.4)$ & & \\
\hline No & $193(49.5)$ & $188(97.4)$ & $5(2.6)$ & & \\
\hline \multicolumn{4}{|c|}{ Having savings (at the bank or elsewhere) } & 267.7 & $0.001^{*}$ \\
\hline Yes & $168(43.1)$ & $4(2.4)$ & 164(97.6) & & \\
\hline No & $222(56.9)$ & $191(86)$ & $31(14)$ & & \\
\hline Systolic blood pressure (mmHg) & $121 \pm 52$ & $120 \pm 73$ & $122 \pm 11$ & 0.36 & 0.71 \\
\hline Diastolic blood pressure $(\mathrm{mmHg})$ & $84 \pm 11$ & $84 \pm 13$ & $84 \pm 9$ & 0.45 & 0.65 \\
\hline
\end{tabular}

TABLE 2: The difference in body composition between the patients and healthy controls.

\begin{tabular}{|c|c|c|c|c|c|}
\hline Body composition & Range score (unit) & $\begin{array}{c}\text { Schizophrenia } \\
\text { Mean (SD) }\end{array}$ & $\begin{array}{c}\text { Control } \\
\text { Mean (SD) }\end{array}$ & $t$-test & $p$ value \\
\hline Height & $\mathrm{cm}$ & $161(8.2)$ & $166(6.2)$ & 6.23 & 0.001 \\
\hline Weight & $\mathrm{kg}$ & $60.6(11.9)$ & $68.8(12.7)$ & 6.61 & 0.001 \\
\hline Body mass index & $\mathrm{kg} / \mathrm{m}^{2}$ & $23.2(4.9)$ & $25.1(4.2)$ & 4.19 & 0.001 \\
\hline Body fat percentage & $\%$ & $25.1(10.3)$ & $25.5(9.9)$ & 0.4 & 0.68 \\
\hline Muscle mass & $\mathrm{kg}$ & $42.7(6.7)$ & $47.5(9.1)$ & 5.9 & 0.001 \\
\hline Muscle quality score & Point & $64.8(12.9)$ & $65.6(13.1)$ & 0.6 & 0.54 \\
\hline Physique rating & $1-9^{*}$ & $3.6(2.9)$ & $4.1(1.6)$ & 1.5 & 0.11 \\
\hline Bone mass & $\mathrm{kg}$ & $2.4(0.3)$ & $2.7(0.4)$ & 7.6 & 0.001 \\
\hline Visceral fat rating & $1-59^{*}$ & $7.6(4)$ & $8.1(4)$ & 1.2 & 0.2 \\
\hline Basal metabolic rate & kcal & $1285(190)$ & $1461(244)$ & 7.91 & 0.001 \\
\hline Metabolic age & Year & $38(11)$ & $36(9)$ & 2.1 & 0.03 \\
\hline Total body water & $\%$ & $50.5(6.8)$ & $47.7(5.7)$ & 4.2 & 0.001 \\
\hline
\end{tabular}

TABle 3: The difference in body mass index between patients and healthy control group.

\begin{tabular}{|c|c|c|c|c|c|}
\hline Body mass index & $\begin{array}{l}\text { Total } \\
n(\%) \\
\end{array}$ & $\begin{array}{c}\text { Schizophrenia } \\
n(\%) \\
\end{array}$ & $\begin{array}{c}\text { Control } \\
n(\%)\end{array}$ & $x 2$ & $p$ value \\
\hline Underweight & $29(7.4)$ & $20(10.3)$ & $9(4.6)$ & \multirow{4}{*}{$<0.001$} & \multirow{4}{*}{29.85} \\
\hline Normal & $212(54.4)$ & $124(63.5)$ & $88(45.1)$ & & \\
\hline Overweight & $63(16.2)$ & $15(7.7)$ & $48(24.6)$ & & \\
\hline Obese & $86(22)$ & $36(18.5)$ & $50(25.7)$ & & \\
\hline
\end{tabular}


TABle 4: Multiple regression analysis for the detailed body composition.

\begin{tabular}{|c|c|c|}
\hline Variable & $\beta$-Coefficient & $p$ value \\
\hline \multicolumn{3}{|l|}{ Body fat } \\
\hline Gender & -10.16 & $<0.001$ \\
\hline Regular exercise & -2.2 & 0.018 \\
\hline BMI & 1.32 & $<0.001$ \\
\hline \multicolumn{3}{|l|}{ Muscle mass } \\
\hline Gender & 11.16 & $<0.001$ \\
\hline Regular exercise & 2.47 & 0.004 \\
\hline Schizophrenia & -3.39 & $<0.001$ \\
\hline BMI & 0.73 & $<0.001$ \\
\hline \multicolumn{3}{|l|}{ Muscle quality } \\
\hline Age & -0.18 & 0.031 \\
\hline Regular exercise & 5.43 & 0.003 \\
\hline BMI & 0.29 & 0.034 \\
\hline \multicolumn{3}{|l|}{ Physique rating } \\
\hline Regular exercise & 0.92 & 0.029 \\
\hline BMI & -0.13 & $<0.001$ \\
\hline \multicolumn{3}{|l|}{ Bone mass } \\
\hline Gender & 0.49 & $<0.001$ \\
\hline Regular exercise & 0.10 & 0.038 \\
\hline Schizophrenia & -0.2 & $<0.001$ \\
\hline BMI & 0.04 & $<0.001$ \\
\hline \multicolumn{3}{|l|}{ Visceral fat } \\
\hline Age & 0.11 & $<0.001$ \\
\hline Gender & 2.73 & $<0.001$ \\
\hline Regular exercise & -0.71 & 0.027 \\
\hline Weight & 0.07 & $<0.001$ \\
\hline BMI & 0.52 & $<0.001$ \\
\hline \multicolumn{3}{|l|}{ Basal metabolic rate } \\
\hline Gender & 250.97 & $<0.001$ \\
\hline Schizophrenia & -26.14 & $<0.001$ \\
\hline BMI & 25.63 & $<0.001$ \\
\hline \multicolumn{3}{|l|}{ Metabolic age } \\
\hline Age & 0.68 & $<0.001$ \\
\hline Gender & -6.01 & $<0.001$ \\
\hline Schizophrenia & 1.83 & 0.011 \\
\hline BMI & 1.05 & $<0.001$ \\
\hline \multicolumn{3}{|l|}{ Total body water } \\
\hline Gender & 4.29 & $<0.001$ \\
\hline BMI & -0.69 & $<0.001$ \\
\hline Schizophrenia & 1.47 & 0.005 \\
\hline
\end{tabular}

body composition. Positive correlations between BMI score and body fat percentage and visceral fat score confirm that as the BMI increases, the other two variables also increase. The increased BMI in patients with schizophrenia is the consequence of antipsychotic medications, psychosocial and socioeconomic risk factors, and an unhealthy lifestyle [27]. Behavioral interventions that control the nutritional intake and encourage regular exercise are proposed, along with other relevant interventions during hospitalization. As such, this study also highlights the importance of regular exercise for improving the body composition of the study subjects.

A significant decrease in bone mass among the patients' group was found. This is associated with female gender, irregular or no exercise, lower BMI, and the diagnosis of schizophrenia. Low bone mineral density is common in patients with schizophrenia [28, 29], and antipsychotic medication is one of the risk factors [29]. However, reports have also indicated that long-term clozapine may protect bone density [30, 31]. Regular exercise also increases bone mass in female adolescents [32]. Further studies might consider whether providing more physical activity and other possible interventions could improve patients' bone mineral density.

This study has several limitations. First, it was conducted during the COVID-19 outbreak. Due to the social distancing policy implemented by the local government, a limited number of patients and healthy controls were included in the study. Second, only hospitalized patients were included, leaving outpatients or those living in the community but using antipsychotics unexamined. Third, several important information such as the age of onset, duration of illness, symptom severity, functional level, and medication was not collected from the patients. Last, the reference group was only matched with gender and not age. Future researchers might consider collecting the abovementioned information from the patients, matching for both age and gender, and including outpatients with schizophrenia or those living in the community in their studies.

\section{Conclusion}

This study highlighted several issues. First, cases of underweight still prevail in this population, despite the rate being lower than previously reported. Second, the diagnosis of schizophrenia is associated with poorer body composition compared to healthy subjects. Lastly, physical activity should be proposed to patients as part of daily treatment during hospitalization.

\section{Data Availability}

The data that support the findings of this study are available on request from the corresponding author.

\section{Disclosure}

The funding source has no implications for the conduct and the result reported in this study.

\section{Conflicts of Interest}

The authors declare no conflict of interest to disclose.

\section{Authors' Contributions}

MS, MA, HS, and JN wrote the protocol and monitored the data collection. RD, RN, and DR conducted the data collection and data analysis. MS performed the data analysis and wrote the initial manuscript. YA contributed to the critical 
review of the manuscript. All authors have approved the final version of the article.

\section{Acknowledgments}

The authors wish to thank the participants of this study. The study was funded by the LPPM of Universitas Syiah Kuala. The work was supported by an award grant to Dr. Marthoenis, Ms. Martina, and Ms. Hasmila Sari by the Institute for Research and Community Services (LPPM), Universitas Syiah Kuala.

\section{References}

[1] A. Annamalai, U. Kosir, and C. Tek, "Prevalence of obesity and diabetes in patients with schizophrenia," World Journal of Diabetes, vol. 8, no. 8, pp. 390-396, 2017.

[2] F. B. Dickerson, C. H. Brown, J. A. Kreyenbuhl et al., "Obesity among individuals with serious mental illness," Acta Psychiatrica Scandinavica, vol. 113, no. 4, pp. 306-313, 2006.

[3] I. M. Cameron, R. J. Hamilton, G. Fernie, and S. A. MacGillivray, "Obesity in individuals with schizophrenia: a case controlled study in Scotland," BJPsych open, vol. 3, no. 5, pp. 254-256, 2017.

[4] K. C. Sung, W. S. Jeong, S. H. Wild, and C. D. Byrne, "Combined influence of insulin resistance, overweight/obesity, and fatty liver as risk factors for type 2 diabetes," Diabetes Care, vol. 35, no. 4, pp. 717-722, 2012.

[5] V. Kotsis, K. Tsioufis, C. Antza et al., "Obesity and cardiovascular risk," Journal of Hypertension, vol. 36, no. 7, pp. 14411455, 2018.

[6] V. A. Chouinard, S. M. Pingali, G. Chouinard et al., "Factors associated with overweight and obesity in schizophrenia, schizoaffective and bipolar disorders," Psychiatry research, vol. 237, pp. 304-310, 2016.

[7] Q. Li, X. Du, Y. Zhang et al., “The prevalence, risk factors and clinical correlates of obesity in Chinese patients with schizophrenia," Psychiatry Research, vol. 251, pp. 131-136, 2017.

[8] V. Mazereel, J. Detraux, D. Vancampfort, R. van Winkel, and M. De Hert, "Impact of psychotropic medication effects on obesity and the metabolic syndrome in people with serious mental illness," Frontiers in Endocrinology, vol. 11, no. October, pp. 1-10, 2020.

[9] N. Sugawara, N. Yasui-Furukori, S. Tsuchimine et al., "Body composition in patients with schizophrenia: comparison with healthy controls," Annals of general psychiatry, vol. 11, no. 1, p. 11, 2012.

[10] J. K. Sharpe, N. M. Byrne, T. J. Stedman, and A. P. Hills, "Bioelectric impedance is a better indicator of obesity in men with schizophrenia than body mass index," Psychiatry Research, vol. 159, no. 1-2, pp. 121-126, 2008.

[11] U. G. Kyle, I. Bosaeus, A. D. De Lorenzo et al., "Bioelectrical impedance analysis-part I: review of principles and methods," Clinical Nutrition, vol. 23, no. 5, pp. 1226-1243, 2004.

[12] R. B. Mazess, H. S. Barden, J. P. Bisek, and J. Hanson, "Dualenergy X-ray absorptiometry for total-body and regional bone-mineral and soft-tissue composition," The American Journal of Clinical Nutrition, vol. 51, no. 6, pp. 1106-1112, 1990.

[13] M. Marra, R. Sammarco, A. De Lorenzo et al., "Assessment of body composition in health and disease using bioelectrical impedance analysis (BIA) and dual-energy X-ray absorptiometry (DXA): a critical overview," Contrast Media \& Molecular Imaging, vol. 2019, article 3548284, pp. 1-9, 2019.

[14] S. E. Saarni, S. I. Saarni, M. Fogelholm et al., "Body composition in psychotic disorders: a general population survey," Psychological medicine, vol. 39, no. 5, pp. 801-810, 2009.

[15] A. Wysokiński and I. Kłoszewska, “Assessment of body composition using bioelectrical impedance in patients with schizophrenia - preliminary report," Archives of Psychiatry and Psychotherapy, vol. 16, no. 1, pp. 31-37, 2014.

[16] S. C. Park, A. Tripathi, A. Avasthi et al., "Relationship between body mass index and extrapyramidal symptoms in Asian patients with schizophrenia: the Research on Asian Psychotropic Prescription Patterns for Antipsychotics (REAP-AP)," Psychiatria Danubina, vol. 32, no. 2, pp. 176-186, 2020.

[17] N. Susilowati, D. Hanim, and Y. L. R. Dewi, "Body mass index of schizophrenic patients with combined antipsychotic therapy," International Journal of Nutrition Sciences, vol. 5, no. 1, pp. 7-12, 2020.

[18] M. Marthoenis, M. Aichberger, I. Puteh, and M. SchoulerOcak, "Low rate of obesity among psychiatric inpatients in Indonesia," International Journal of Psychiatry in Medicine, vol. 48, no. 3, pp. 175-183, 2014.

[19] S. W. Woods, "Chlorpromazine equivalent doses for the newer atypical antipsychotics," The Journal of Clinical Psychiatry, vol. 64, no. 6, pp. 663-667, 2003.

[20] Tanita Corp, Tanita RD 953 Intruction Manual, 2020.

[21] Tanita Corp, "Time to find out what you're made of ! [Internet]," 2022, https://tanita.eu/help-guides/understandingyour-measurements/.

[22] StataCorp, Stata Statistical Software: Release 13, StataCorp LP, Collage Station, TX, 2013.

[23] B. Konarzewska, E. Stefańska, A. Wendołowicz et al., "Visceral obesity in normal-weight patients suffering from chronic schizophrenia," BMC Psychiatry, vol. 14, no. 1, pp. 1-9, 2014.

[24] R. N. Baumgartner, D. L. Waters, D. Gallagher, J. E. Morley, and P. J. Garry, "Predictors of skeletal muscle mass in elderly men and women," Mechanisms of Ageing and Development, vol. 107, no. 2, pp. 123-136, 1999.

[25] N. Sugawara, K. Maruo, T. Sugai et al., "Prevalence of underweight in patients with schizophrenia: a meta-analysis," Schizophrenia Research, vol. 195, pp. 67-73, 2018.

[26] S. Yim Loh, A. Yusof, and A. Kadir Abu Bakar, "Visceral obesity in the normal-weight people-comparing chronic schizophrenia and healthy control subjects," Brain and Nerves, vol. 1, no. 2, pp. 1-6, 2017.

[27] P. Manu, L. Dima, M. Shulman, D. Vancampfort, M. De Hert, and C. U. Correll, "Weight gain and obesity in schizophrenia: epidemiology, pathobiology, and management," Acta Psychiatrica Scandinavica, vol. 132, no. 2, pp. 97-108, 2015.

[28] P. T. Tseng, Y. W. Chen, P. Y. Yeh, K. Y. Tu, Y. S. Cheng, and C. $\mathrm{K} . \mathrm{Wu}$, "Bone mineral density in schizophrenia an update of current meta-analysis and literature review under guideline of PRISMA," Medicine, vol. 94, no. 47, article e1967, 2015.

[29] C. Y. Chen, H. Y. Lane, and C. H. Lin, "Effects of antipsychotics on bone mineral density in patients with schizophrenia: gender differences," Clinical Psychopharmacology and Neuroscience, vol. 14, no. 3, pp. 238-249, 2016.

[30] C. H. Lin, C. Y. Lin, H. S. Wang, and H. Y. Lane, "Long-term use of clozapine is protective for bone density in patients with schizophrenia," Scientific Reports, vol. 9, no. 1, pp. 1-7, 2019. 
[31] C. H. Lin, K. H. Huang, Y. C. Chang et al., "Clozapine protects bone mineral density in female patients with schizophrenia," The International Journal of Neuropsychopharmacology, vol. 15, no. 7, pp. 897-906, 2012.

[32] T. Okayama, K. Kinouchi, and H. Watanabe, "Lifestyle and environmental factors affecting bone mass in Japanese female adolescents," Journal of Pediatric Nursing, vol. 56, pp. e55e61, 2021. 